\title{
Estrogen inhibits the overgrowth of Escherichia coli in the rat intestine under simulated microgravity
}

\author{
YONGTAO YANG $^{1 *}$, CHANGMIN QU $^{1 *}$, SHUWEN LIANG $^{1}$, GANG WANG $^{2}$, HAOLUN HAN $^{2}$, NA CHEN $^{2}$,

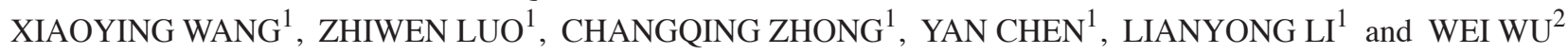 \\ Departments of ${ }^{1}$ Gastroenterology and ${ }^{2}$ Otorhinolaryngology, \\ The 306th Hospital of PLA, Beijing 100101, P.R. China
}

Received June 15, 2017; Accepted November 13, 2017

DOI: $10.3892 / \mathrm{mmr} .2017 .8109$

\begin{abstract}
Microgravity can affect many aspects of intestinal homeostasis, leading to an increased risk of colitis. Estrogen, the most frequently affected hormone when under simulated microgravity, regulates the permeability of the colonic mucosa barrier. The associations between alterations in intestinal microbiota and increased susceptibility under microgravity have not been thoroughly elucidated. The aim of the present study was to evaluate the changes in intestinal microbiota under simulated microgravity and to investigate the protective effect of estrogen against those changes. The hindlimb unweighting (HU) model was used to simulate microgravity in rats. Estrogen was administered via intramuscular injection. Amplicons of the V3 variable regions of bacterial 16S rDNA were analyzed using denaturing gradient gel electrophoresis (DGGE), cloning and sequencing. Several specific bacterial groups were assayed using quantitative-polymerase chain reaction. Bacterial translocation was evaluated by detecting serum lipopolysaccharide (LPS) and LPS binding protein (LBP) levels. DGGE profiles generated by universal primers revealed minor, though specific, changes in bacterial communities under simulated microgravity, particularly the band matching the sequence of Escherichia coli (E. coli). The quantification of 16S RNA revealed increased numbers of Bacteroides fragilis, E. coli and Fusobacterium nucleatum; however, Bifidobacteria longum significantly decreased under microgravity. Estrogen inhibited the overgrowth of E. coli, and decreased the levels
\end{abstract}

Correspondence to: Dr Lianyong Li, Department of Gastroenterology, The 306th Hospital of PLA, 9 Anxiang Beili, Beijing 100101, P.R. China

E-mail: lilianyong001@163.com

Professor Wei Wu, Department of Otorhinolaryngology, The 306th Hospital of PLA, 9 Anxiang Beili, Beijing 100101, P.R. China

E-mail: cherytmmu@sina.com

*Contributed equally

Key words: microbiota, microgravity, estrogen, Escherichia coli of LBS and LBP under simulated microgravity. These results demonstrated that simulated microgravity alters the intestinal microflora and may contribute to bacterial translocation in the gut mucosa. The data also suggested that further investigations evaluating the administration of estrogen to protect against microgravity-associated diseases may be required.

\section{Introduction}

Microgravity has a profound effect on the physiology of humans, leading to increased susceptibility to many diseases (1). Both spaceflight and ground-based human and animal studies have reported many physiological adaptations to microgravity in immune and endocrine systems (2). The major endocrine changes associated with exposure to hypogravity include disrupted reproductive cycles. For example, microgravity simulated using a hindlimb suspension (HLS) model in rats demonstrated lengthened estrous cycles and prolonged diestrus due to hypoestrogenism (3). It has in recent years been established that the gut microbiota play a crucial role in the maturation of the immune and endocrine systems. Mechanisms through which microbiota mediate these functions include the cometabolism of steroid hormones and low-molecular weight dietary compounds with hormone-like activities that regulate the immune system (4). This microbial/hormonal interplay is bidirectional, stress-induced neuroendocrine hormones have been observed to increase bacterial adhesion to host tissues and alter the growth and virulence of bacteria through the regulation bacterial gene expression (4).

Intestinal microbiota and intestinal mucous barrier both respond to simulated microgravity and contribute to the susceptibility to inflammation in the gut microenvironment (5). Simulated microgravity has been reported to result in a transient increase in circulating LPS and a stimulation of the innate immune system (6). Simulated microgravity disrupts intestinal microflora and the innate immune system, leading to a proinflammatory shift in the gut microenvironment and an increase in colitis susceptibility (7). Estrogen, the most prominent affected hormone under simulated microgravity, regulates the permeability of the colonic mucosa barrier $(3,8)$. Estrogen gene knock-out mice have been shown to present with many prepathogenic phenotypes, including abnormal 
colonic histology and disrupted cellular tight junctions (9). These architectural abnormalities facilitate the invasion of intestinal bacteria, resulting in localized infection and enhanced levels of colonic inflammation (10). However, there remains little knowledge regarding the effects of estrogen on the composition of the gut microbiota and their downstream influences on intestine barrier dysfunction.

Studies have found that the alteration of even a single host gene can significantly change host-driven selective pressures that lead to changes in the structure and function of the commensal gastrointestinal microbiota $(9,11,12)$. We thus sought to characterize the possible impact of simulated hypogravity on gut commensal microbiota and to evaluate the protective effect of estrogen on the homeostasis of intestinal microbiota in rats under simulated hypogravity. The predominant fecal microbiota was analyzed using two universal primers targeting the V3 regions of the 16S rRNA gene. Specific subpopulations were quantified with genusand group-specific primers to complement the analysis and interpretation of the results obtained with the universal primers. The study was helpful to more thoroughly evaluate the potential role of hypogravity in the pathogenesis of gastrointestinal diseases in humans.

\section{Materials and methods}

Animals and treatment. The animals were treated as previously described (13). Briefly, Male Wistar rats (Eight-week-old, approximately $250 \mathrm{~g}$ ) were purchased from the Experimental Animal Center, Academy of Military Medical Sciencs. The mice were kept in a specific pathogen-free animal facility at the State Key Laboratory of Space Medicine Fundamentals. All experiments were performed in accordance with the 'Guide for the Care and Use of Laboratory Animals' published by the US NIH (National Institutes of Health Publication No. 85-23, revised 1996) and were approved by the Committee on the Ethics of Animal Experiments of the 306th Hospital of the PLA. The rats were caged separately in a room maintained at $21^{\circ} \mathrm{C}$ and controlled light/dark cycles $(12 / 12 \mathrm{~h})$. The rats were randomly assigned to four groups of 10 rats each as follows: A control group (without any treatment), a simulated microgravity group (tail suspension for 8 weeks group) and simulated microgravity combined with estrogen for 4 weeks and 8 weeks groups (estrogen was administered via the intramuscular injection of estradiol benzoate at a dose of $80 \mu \mathrm{g} / \mathrm{Kg}$ daily for first seven days, then rat were euthanized at the timepoints of 4 weeks and 8 weeks, respectively). To achieve simulated microgravity, individual rats were subjected to hindlimbs suspension. The technique of hindlimbs suspension was performed by the use of a tail harness that partially elevated the hindlimbs above the floor of the cage according to a previously published method (14). Briefly, the tail was firstly fixed with an adhesive tape. Then the tail was suspended via a tether connecting to a horizontal tube at the top of the cage. The upper end of the tether included a small pulley, which allowed the rat rolled freely along the length of the tube. The animals were maintained in 30 headdown tilt position with the hindlimbs elevated $0.5 \mathrm{~cm}$ above the floor when fully extended. The animals were euthanized under anesthesia.
Samples and DNA extraction. After the four-week or eight-week tail suspension, the rats were anesthetized with $1 \%$ sodium pentobarbital $(45 \mathrm{mg} / \mathrm{kg})$. Fecal pellets were collected in tubes and weighted. Samples were quickly frozen in liquid nitrogen and stored at $80^{\circ} \mathrm{C}$ until nucleic acid extraction was performed. Fecal bacteria DNA was extracted using the modified CTAB method as previously described and stored at $80^{\circ} \mathrm{C}(15)$. DNA integrity was analyzed by loading $2 \mu 1$ DNA on a $1 \%$ agarose gel stained with ethidium bromide. The concentration and purity of the extracted DNA were analyzed using ultraviolet absorption at 260/280 and 230/280 $\mathrm{nm}$ ratios.

PCR amplification and DGGE analysis. PCR amplification and DGGE analysis were performed as previously described (16). Briefly, the variable V3 region of the $16 \mathrm{~S}$ rRNA gene was amplified using the universal bacterial primers F357-GC and R518 with the sequences of 5'-CCG AATTCGTCGACAACAGAGTTTGATCCTGGCTCAG-3' and 5'-CCCGGGATCCAAGCTTACGGCTACCTTGTT ACGACTT-3', respectively. The PCR was performed as previously described. The amplified sequences of $16 \mathrm{~S}$ rRNA were analyzed via DGGE fingerprinting analysis using 35 to $70 \%$ denaturing gel. Each lane uploaded $30 \mu \mathrm{l}$ of PCR product, and electrophoresis was performed at $70 \mathrm{~V}$ for $990 \mathrm{~min}$. Next, the DGGE gels were stained for $30 \mathrm{~min}$ with ethidium bromide ( $50 \mu \mathrm{g}$ in $500 \mathrm{ml} 1 \mathrm{X}$ TAE buffer), and the band profiles were visualized and analyzed with a Quantity One Analysis System (Bio-Rad, California, USA). In short, all fingerprinting profiles were aligned using the reference lanes and then compared. The numeric value of the relative intensity per band class was then exported for all profiles and further analyzed. The Berger-Parker index, which was used to assess species richness, and $\alpha$ diversity measures, including the Shannon and Simpson diversity indices, were calculated based on the band profiles according to previously described method (17).

Cloning of the PCR-amplified products and sequence analysis. PCR products were extracted from the band using an Invitrogen PCR product purification kit (Invitrogen Life Technologies, Shanghai, China) and cloned in E. coli JM109 using the pGEM-T vector system (15). Colonies of ampicillin-resistant transformants were selected and transferred to Luria broth medium. After incubation at $37^{\circ} \mathrm{C}$ overnight, one hundred microliters of cultures were recovered and dissolved in $10 \mathrm{ml}$ TE buffer. The solutions were then boiled to lyse the cells. The cell lysates were used as templates for PCR reactions using the pGEM-T-specific primers Sp6 and T7 to verify the size of the DNA inserts (15). The plasmids with appropriately sized inserts were sent for sequencing (Invitrogen Life Technologies). Homology searches of the GenBank DNA database were performed with the BLAST Search Tool. A phylogenetic tree was then determined via MEGA4 software based on the neighbor-joining algorithm and the Jukes-Cantor model to demonstrate the evolutionary relationship (18).

Real-time PCR assay. Significant differences of intensity of the bacterial species after DGGE analysis were confirmed using real-time PCR. The abundance of specific intestinal bacterial 
Table I. Bacterial group-specific primers used in reverse transcription-polymerase chain reaction for microbiota studies.

\begin{tabular}{ll}
\hline Group & \multicolumn{1}{c}{ Primer sequences (5'-3') } \\
\hline Eubacteria & F-ACTCCTACGGGAGGCAGCAGT \\
(all bacteria) & R-ATTACCGCGGCTGCTGGC \\
Escherichia & F-GACCTCGGTTTAGTTCACAGA \\
coli & R-CACACGCTGACGCTGACCA \\
Bifidobacterium & F-GCCGTATCTCTACGACCGTCG \\
longum & R-TATCGGGGAGCAAGCGAGAG \\
Bacteroides & F-ATAGCCTTTCGAAAGRAAGAT \\
fragilis & R-CCAGTATCAACTGCAATTTTA \\
Fusobacterium & F-GGATTTATTGGGCGTAAAGC \\
nucleatum & R-GGCATTCCTACAAATATCTACGAA \\
\hline
\end{tabular}

F, forward; R, reverse.

groups chosen from four representative phyla were measured via $\mathrm{qPCR}$ using group-specific $16 \mathrm{~S}$ rRNA gene primers. All primers used in this study are listed in Table I. The qPCR assay was performed as previously described (19). Briefly, the qPCR assay was performed with a SYBR Premix Ex Taq (Takara Bio, Inc., Otsu, Japan) on an Applied Biosystems 7500 fast real-time PCR system (Applied Biosystems, Ghent, Belgium). For each primer set, a constructed plasmid was chosen to create a 10-log fold standard curve to directly quantify all samples. Each qPCR contained $10 \mu \mathrm{l}$ SYBR Premix Ex Taq, $0.4 \mu \mathrm{l}$ of a $10 \mu \mathrm{mol} / 1$ $\mathrm{F} / \mathrm{R}$ primer mix, and $1 \mu \mathrm{l}$ of the respective template DNA. Amplifications were performed under the following temperature profiles: One cycle at $95^{\circ} \mathrm{C}$ for $3 \mathrm{~min}, 40$ cycles of denaturation at $95^{\circ} \mathrm{C}$ for $30 \mathrm{sec}$, annealing for $40 \mathrm{sec}$, and extension for $30 \mathrm{sec}$. Fluorescence was measured after the extension phase of each cycle at an appropriate temperature for $10 \mathrm{sec}$ to avoid interference from primer dimers, secondary structure, or spurious priming. A final extension step was sustained for $5 \mathrm{~min}$.

Lipopolysaccharide (LPS) and LPS binding protein (LBP) detection. Blood was collected via cardiac puncture. LPS and LBP detection in the serum were based on a previously performed method (20). Circulating endotoxin (LPS) was analyzed using a Limulus Amoebocyte Lysate (LAL) assay QCL-1000 (Lonza AG, Valais Switzerland) in duplicate in 96-well plates according to the manufacturer's instructions; lipopolysaccharide-binding protein (LBP) levels were detected using the LBP Elisa kit (Abnova, Taipei, Taiwan). The lower limit of detection for each assay is $0.1 \mathrm{EU} / \mathrm{ml}$ for LPS and $5 \mathrm{ng} / \mathrm{ml}$ for LBP.

Statistical analysis. Differences between the treatment and control groups were compared regarding band intensity and bacterial count via RT-PCR between the different groups for significance testing with an ANOVA program. Statistically significant differences between 2 groups were evaluated using a 2-tailed unpaired Student's t test. All values are expressed as the means \pm SDs of replicates. Two-sided P-values $<0.05$ were considered to indicate a statistically significant difference. The statistical analysis of the results was performed via SigmaPlot software (Systat Software Inc., San Jose, California, USA).
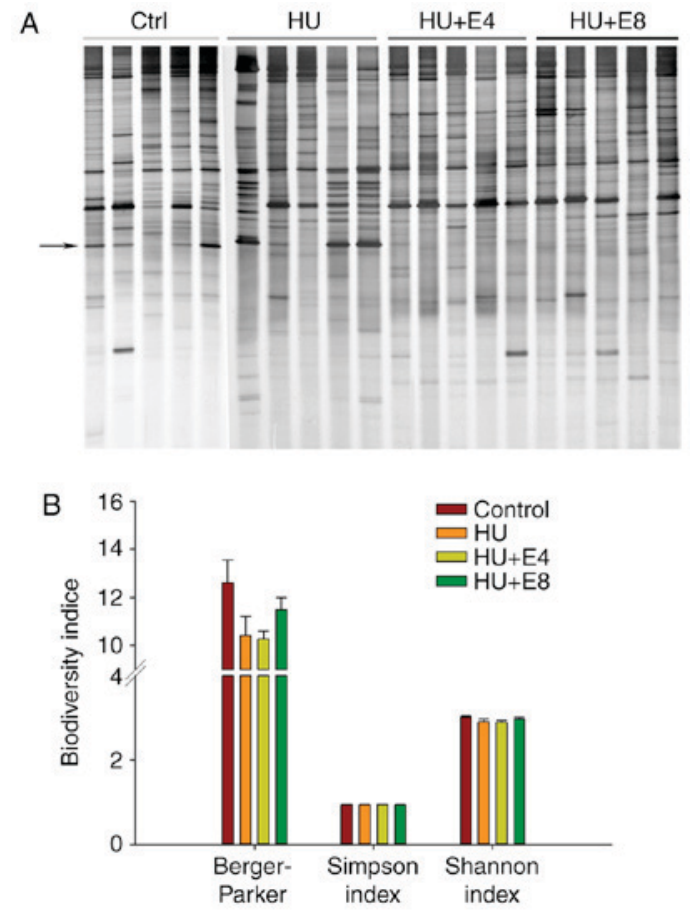

Figure 1. DGGE profiles showed microbial diversity in the rat feces of different groups. (A) The figure shows DGGE gels of the V3 hypervariable 16S rDNA region, demonstrating the microbiota's composition in the feces of rats from control (Ctrl), simulated microgravity (HU), simulated microgravity combined with estrogen for 4 weeks (HU+E4) and simulated microgravity combined with estrogen for 8 weeks (HU+E8). The arrow indicates the most significantly changed band. Number indicated the bands that were cloned and sequenced. (B) Comparison of biodiversity indices of feces microbiota between the control and treatment groups. There were no significant difference between groups (P-value were $0.09,0.25,0.17$ for Berger-parker, Simpson index, Shannon index, respectively). Bars indicate the standard deviation of five samples in the same group. DGGE, denaturing gradient gel electrophoresis; HU, hindlimb unweighting.

\section{Results}

PCR-DGGE analysis of changes in fecal bacterial populations associated with simulated microgravity and estrogen exposure. DGGE bands from the PCR products of the $\mathrm{V} 3$ regions of $16 \mathrm{~S}$ rRNA genes from rat fecal samples of control, simulated microgravity or simulated microgravity combined with estrogen administration groups are shown in Fig. 1A. Most bands appeared to be unaffected by simulated microgravity exposure or estrogen administration. To obtain an objective evaluation of the DGGE profiles of the various groups, the electrophoretic bands underwent numerical analysis based on band density. The beta diversity measures included Shannon and Simpson indices, which were computed to compare the diversity of the dominant bacterial microbiota in the rat feces of the control and treatment groups. The Berger-Parker index, which reflects species richness, was also calculated to compare the discrepancy between groups. A statistical analysis showed that the diversity indices did not significantly differ between the groups (Fig. 1B). However, species richness was slightly lower after exposure to simulated microgravity.

Sequencing of DGGE bands and predominant gut microflora change. To further identify the predominant populations, 


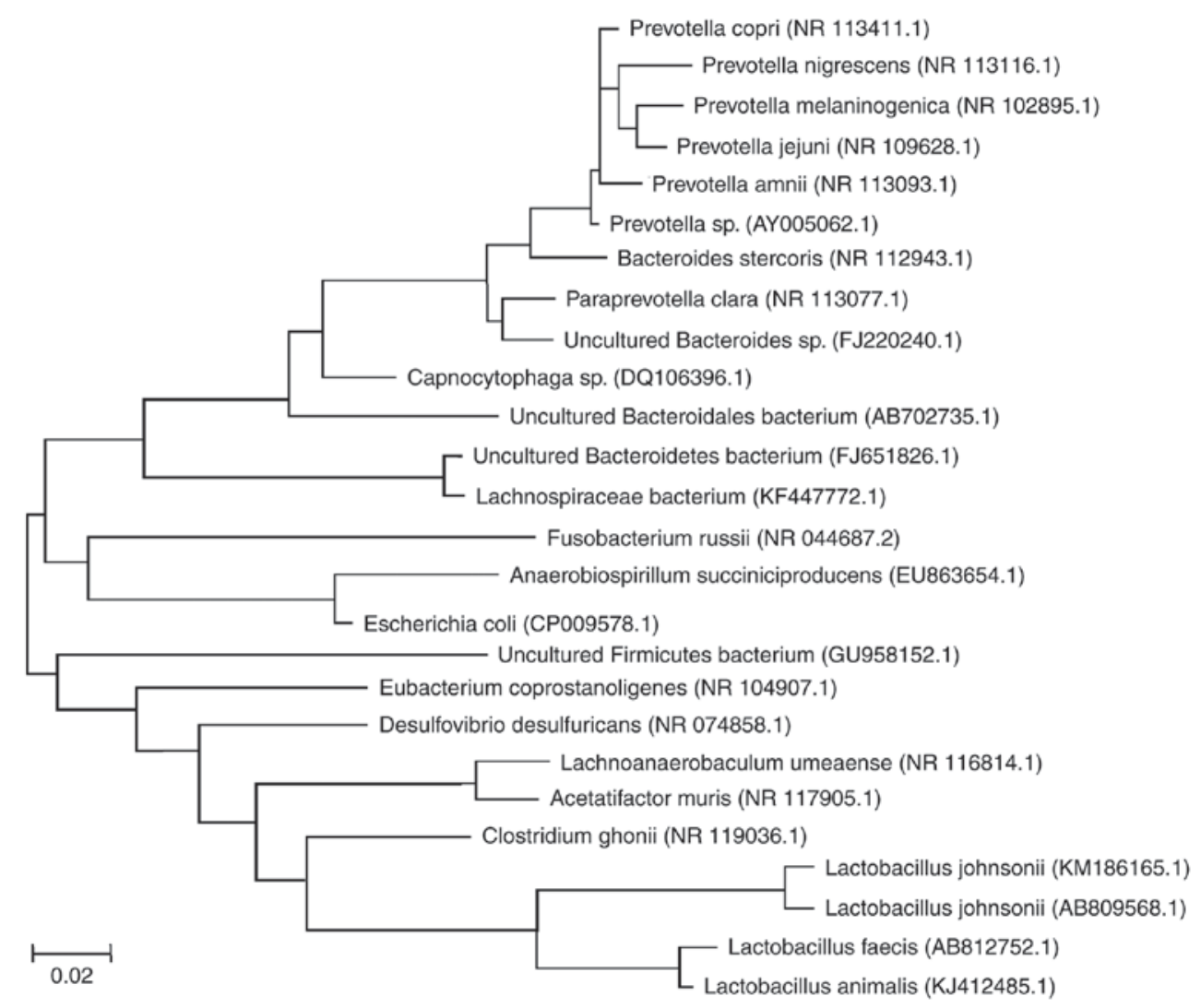

Figure 2. Phylogenetic tree constructed from the sequences of clones derived from bands of the V3 region of the 16S rRNA gene in the DGGE. Evolutionary distances were constructed using the neighbor-joining algorithm and the Jukes-Cantor model. Of note, only the representive aligned bacteria species were demonstrated, specific points that showed the differences between clones were omitted (refer to supporting information for full version of Phylogenetic tree). DGGE, denaturing gradient gel electrophoresis.

36 bands of the 16S rRNA genes from rat feces samples were extracted from the gel, and three independent clones were constructed and subjected to DNA sequencing for each band. The sequences of these clones were compared with the NCBI database, and representative aligned bacteria species were demonstrated in the brief phylogenetic tree as shown in Fig. 2. Overall, the two major phyla (Firmicutes and Bacteroidetes) had similar abundance in all four groups, whereas there were changes in the abundance of the Proteobacteria and Fusobacteria phyla (Fig. 3A and Table II). Additionally, treatment with microgravity and estrogen changed the abundance of the colonic microbiota at the genus level (Fig. 3B and Table II). Escherichia coli, which belongs to the Gram-negative Enterobacteriaceae family, showed the most remarkable band change during exposure to simulated microgravity and estrogen. As shown in Fig. 1, the Escherichia coli band was significantly intensified after exposure to microgravity, indicating the overgrowth of Escherichia coli under simulated microgravity. However, estrogen greatly protected the gut microbiota from changes under microgravity in rats.

qPCR to analyze specific bacterial population change. A qPCR assay was used to specifically analyze the abundance of representative bacterial species. The four bacterial species of Bifidobacteria longum (B. longum), Bacteroides fragilis (B. fragilis), Escherichia coli (E. coli) and Fusobacterium nucleatum (F. nucleatum) were selected from Firmicutes, Bacteriodetes, Proteobacteria and Fusobacteria, respectively, based on the findings of specific primers and for being with known functions within the four major phyla. The results were expressed as the estimated average number of $16 \mathrm{~S}$ rRNA genes of the target bacteria species. Because qPCR measures the number of 16S rRNA gene copies per sample and not actual bacterial numbers, these values are directly related and strongly correlated (21). The subgroups of Bacteroides fragilis (B. fragilis), Escherichia coli (E. coli) and Fusobacterium nucleatum ( $F$. nucleatum) increased significantly in number under simulated microgravity, whereas the species of Bifidobacteria longum (B. longum) significantly decreased under this circumstance $(\mathrm{P}<0.05$ for all bacterial groups, Fig. 4). However, the administration of estrogen resulted in a reversion of the microbiota change to that of the control group at intervals of 4 and 8 weeks.

Simulated microgravity and estrogen affected the serum level of LPS and LBP. To evaluate whether simulated microgravity and the administration of estrogen under simulated microgravity affect bacterial translocation, we detected serum LPS and LBP levels as surrogate markers. It is rational that bacterial translocation enhances circulating levels of bacterial components including LPS (7). LPS is released from Gram-negative bacteria in an aggregate form due to its amphiphilic nature. LPS aggregates are converted into monomers by LBP. LBP then transfers LPS monomers to CD14 by binding to the amphipathic lipid A of bacterial LPS, which in turn transfers it to Toll-like receptor (TLR) 4, leading to signaling (7). As 
Table II. Relative distributions of bacterial phylotypes and genera in the rat feces of different groups.

\begin{tabular}{|c|c|c|c|c|}
\hline Groups & Control & $\mathrm{HU}$ & $\mathrm{HU}+\mathrm{E} 4$ & $\mathrm{HU}+\mathrm{E} 8$ \\
\hline \multicolumn{5}{|l|}{ Phylum } \\
\hline $\begin{array}{l}\text { Firmicutes } \\
\text { (range) }\end{array}$ & $\begin{array}{c}61.2 \\
(51.2-69.1)\end{array}$ & $\begin{array}{c}54.4 \\
(36.3-61.5)\end{array}$ & $\begin{array}{c}65.8 \\
(51.5-70.1)\end{array}$ & $\begin{array}{c}62.1 \\
(43.1-69.4)\end{array}$ \\
\hline SEM & 4.9 & 5.9 & 4.2 & 6.3 \\
\hline P-value & & 0.2134 & 0.2514 & 0.1972 \\
\hline $\begin{array}{l}\text { Bacteroidetes } \\
\text { (range) }\end{array}$ & $\begin{array}{c}32.8 \\
(20.9-38.3)\end{array}$ & $\begin{array}{c}29.5 \\
(22.4-37.4)\end{array}$ & $\begin{array}{c}33.4 \\
(27.2-45.7)\end{array}$ & $\begin{array}{c}37.1 \\
(28.6-55.2)\end{array}$ \\
\hline $\begin{array}{l}\text { SEM } \\
\text { P-value }\end{array}$ & 4.5 & $\begin{array}{c}3.4 \\
0.3131\end{array}$ & $\begin{array}{c}5.7 \\
0.4512\end{array}$ & $\begin{array}{c}6.2 \\
0.2578\end{array}$ \\
\hline $\begin{array}{l}\text { Proteobacteria } \\
\quad \text { (range) }\end{array}$ & $\begin{array}{c}5.7 \\
(0.0-13.4)\end{array}$ & $\begin{array}{c}15.5 \\
(0.2-24.7)\end{array}$ & $\begin{array}{c}0.5 \\
(0.0-1.8)\end{array}$ & $\begin{array}{c}0.5 \\
(0.0-1.6)\end{array}$ \\
\hline $\begin{array}{l}\text { SEM } \\
\text { P-value }\end{array}$ & 1.8 & $\begin{array}{c}2.9 \\
0.0035\end{array}$ & $\begin{array}{c}0.21 \\
0.0075\end{array}$ & $\begin{array}{c}0.17 \\
0.0067\end{array}$ \\
\hline $\begin{array}{l}\text { Fusobacteria } \\
\text { (range) }\end{array}$ & $\begin{array}{c}0.3 \\
(0.0-1.2)\end{array}$ & $\begin{array}{c}0.6 \\
(0.0-2.4)\end{array}$ & $\begin{array}{c}0.3 \\
(0.0-1.4)\end{array}$ & $\begin{array}{c}0.3 \\
(0.0-1.1)\end{array}$ \\
\hline SEM & 0.028 & 0.044 & 0.038 & 0.041 \\
\hline P-value & & 0.15 & 0.47 & 0.43 \\
\hline \multicolumn{5}{|l|}{ Genus } \\
\hline Lactobacillus & 34.4 & 25.7 & 33.3 & 30.5 \\
\hline SEM & 4.5 & 3.4 & 3.5 & 2.7 \\
\hline P-value & & 0.2109 & 0.2732 & 0.3578 \\
\hline Acetatifactor & 1.1 & 1.1 & 0.9 & 1.4 \\
\hline SEM & 0.15 & 0.14 & 0.11 & 0.21 \\
\hline P-value & & 0.1354 & 0.2036 & 0.2677 \\
\hline Lachnoanaerobaculum & 1.2 & 0.6 & 0.8 & 1.6 \\
\hline SEM & 0.15 & 0.07 & 0.12 & 0.23 \\
\hline P-value & & 0.0284 & 0.0439 & 0.2421 \\
\hline Eubacterium & 0.2 & 0.3 & 0.2 & 0.4 \\
\hline SEM & 0.035 & 0.023 & 0.021 & 0.047 \\
\hline P-value & & 0.0522 & 0.4189 & 0.159 \\
\hline Bacteroides & 9.7 & 8.7 & 10.8 & 13.5 \\
\hline SEM & 0.75 & 1.08 & 1.23 & 1.42 \\
\hline P-value & & 0.16 & 0.21 & 0.11 \\
\hline Prevotella & 18.1 & 16.3 & 19 & 14.9 \\
\hline SEM & 1.34 & 1.54 & 1.26 & 0.86 \\
\hline P-value & & 0.127 & 0.138 & 0.058 \\
\hline Capnocytophaga & 1.8 & 1.9 & 1.1 & 1.8 \\
\hline SEM & 0.24 & 0.18 & 0.16 & 0.21 \\
\hline P-value & & 0.38 & 0.26 & 0.31 \\
\hline Escherichia & 5.7 & 15.5 & 0.5 & 0.5 \\
\hline SEM & 0.61 & 3.2 & 0.06 & 0.07 \\
\hline P-value & & 0.0035 & 0.0067 & 0.0075 \\
\hline Fusobacterium & 0.3 & 0.6 & 0.3 & 0.3 \\
\hline SEM & 0.028 & 0.044 & 0.038 & 0.041 \\
\hline P-value & & 0.15 & 0.47 & 0.43 \\
\hline Unclassified & 27.5 & 29.3 & 33.1 & 35.2 \\
\hline SEM & 1.88 & 2.54 & 2.06 & 2.26 \\
\hline P-value & & 0.258 & 0.138 & 0.085 \\
\hline
\end{tabular}

Data are expressed as the mean, range and SEM percentage abundance of the total composition ( $\mathrm{n}=5$ per group). The threshold for statistical significance was set to $\mathrm{P}<0.05$. SEM, standard error mean; HU, hindlimb unweighting. 
A

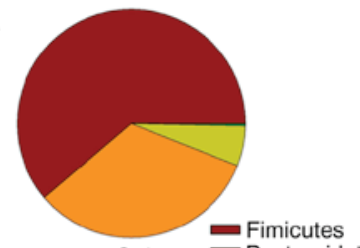

Ctrl Bacteroidetes
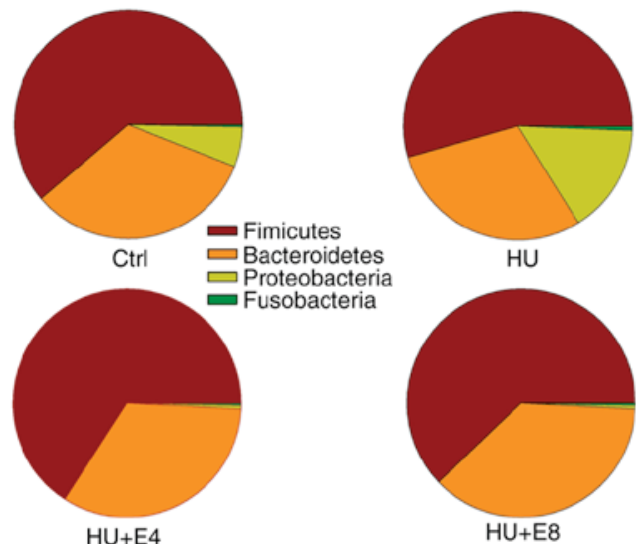

HU
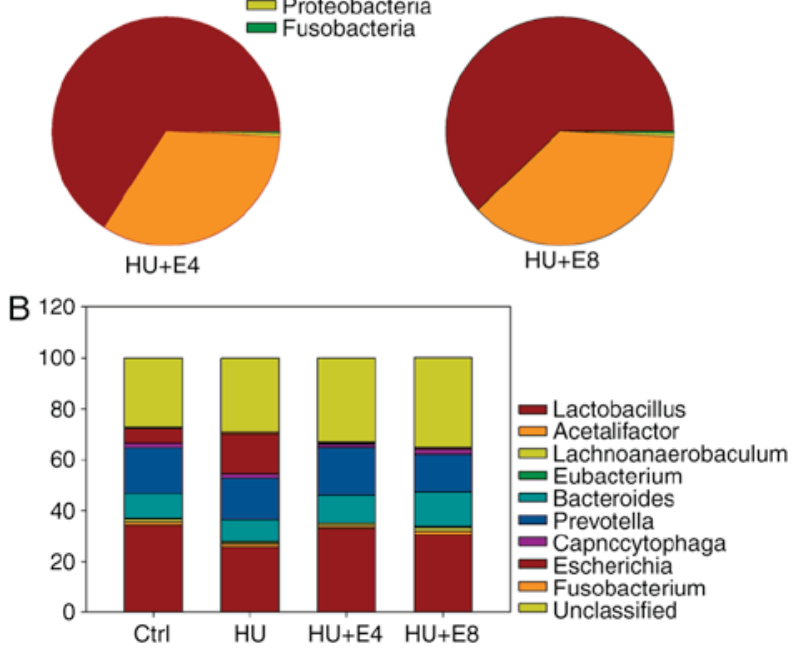

Figure 3. Distribution of bacterial phylotypes (A) and subgroups (B) in the fecal samples of rats from the following groups: Control (Ctrl), simulated microgravity ( $\mathrm{HU})$, simulated microgravity combined with estrogen for 4 weeks (HU+E4) and simulated microgravity combined with estrogen for 8 weeks (HU+E8). 16S rRNA-base analyses were derived from DGGE band intensities. Data are expressed as the mean percentage abundance ( $\mathrm{n}=5$ rats/group). Most of the bacteria belonged to the Firmicutes and the Bacteroidetes, whereas Fusobacteria were very rare. HU, hindlimb unweighting; DGGE, denaturing gradient gel electrophoresis.

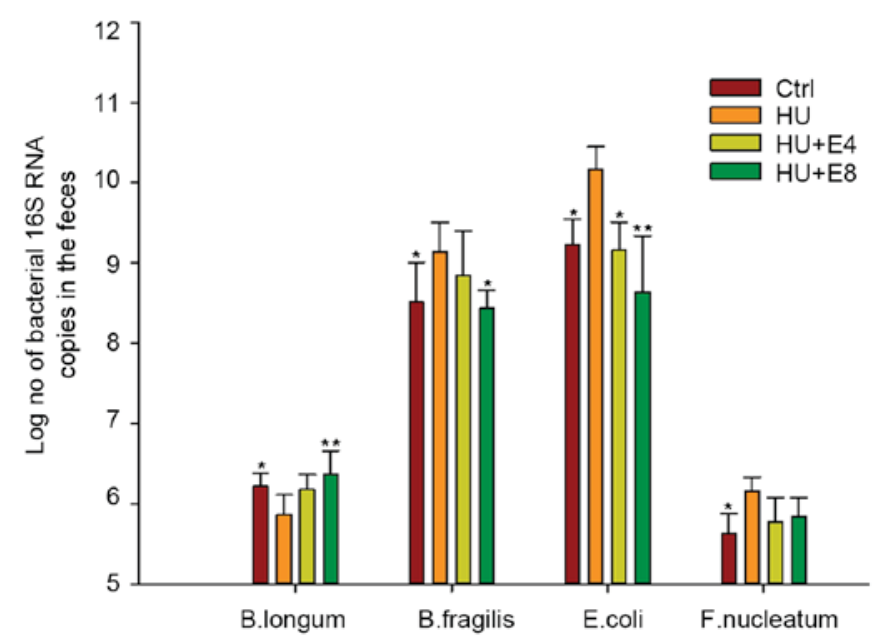

Figure 4. Quantitative-polymerase chain reaction analysis measured the abundance of specific bacterial groups in the control (Ctrl), simulated microgravity (HU), simulated microgravity combined with estrogen for 4 weeks (HU+E4) and simulated microgravity combined with estrogen for 8 weeks (HU+E8) groups. Simulated microgravity and estrogen administration appeared to affect the bacterial counts in the feces of rats. ${ }^{*} \mathrm{P}<0.05$ and ${ }^{* *} \mathrm{P}<0.01$ vs. HU group. HU, hindlimb unweighting.

shown in Fig. 5, both LPS and LPB levels were significantly increased in rats under microgravity; however, a significant reduction was observed when estrogen was administered at 4 and 8 weeks $(\mathrm{P}<0.05)$.
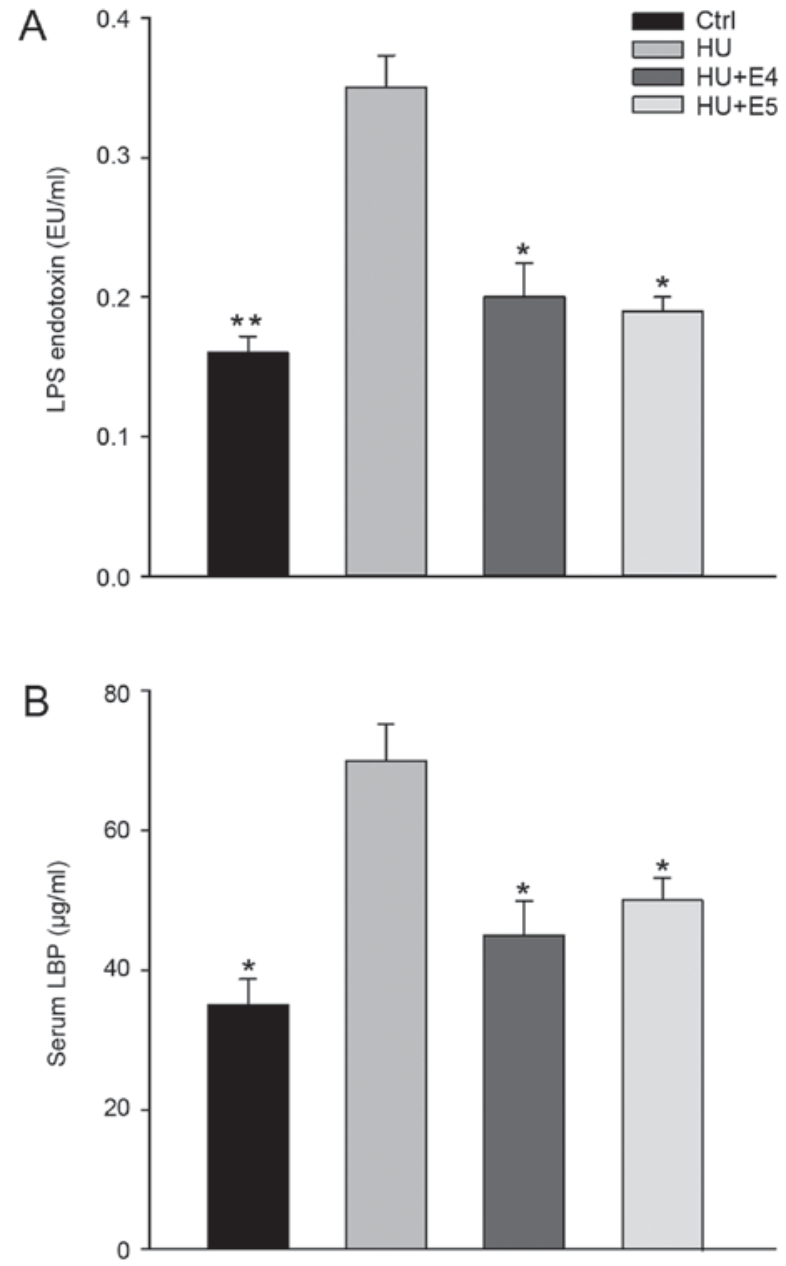

Figure 5. Simulated microgravity increased circulating (A) LPS and (B) LBP levels, whereas estrogen protected against such an increase. Data shown are the averages for five rats per group analyzed in triplicate; error bars are standard error mean. Statistically significant differences were observed for both LPS and LBP levels between different groups. ${ }^{*} \mathrm{P}<0.05$ and ${ }^{* *} \mathrm{P}<0.01$ vs. HU group. LPS, lipopolysaccharide; LBP, LPS binding protein; HU, hindlimb. unweighting.

\section{Discussion}

The long-duration of exposure to the space travel environment exerts huge impacts on human health. Many immune functions have been reported to be compromised during space travel. Spaceflight-associated stresses were shown to compromise the important capability of neutrophils and monocytes to engulf $E$. coli and also reduced their ability to degranulate and elicit an oxidative burst (1). Spaceflight represents a unique environment characterized by decreased gravity. Although immunological and physiological aspects during spaceflight have been extensively studied, little effort has been focused on the intestinal microbiota under microgravity. The present study demonstrated that the DGGE profiles of 16s RNA PCR amplicons changed after exposure to simulated microgravity, suggesting a corresponding change in the rat fecal bacteria flora, although the overall microbiota alpha diversity indices (that is, diversity within a given community), including Shannon's and Simpson's index from the microgravity group, were not statistically significant compared with the control groups. The most significant change at the phyla level occurred for Proteobacteria, which is dominated by an $E$. 
coli population. Although the abundance of dominant intestinal bacteria phyla (such as Firmicutes and Bacteroides) did not significantly change overall, the specific sub-phylum composition of microbiota, such as $B$. fragilis and $F$. nucleatum, were variable, as demonstrated by qPCR with a specific probe.

It has been reported that microgravity-induced decrease in stress on the surface of microorganisms may influence the growth and physiology of bacteria (22). So it would be rational to speculate that the microgravity-induced decrease in tension on the surface of microorganisms may lead to the overgrowth of some species of bacteria, although the exact mechanism need further elucidate. On the other side, the microbiota within the body responds and adapts to changes in its host, such as those induced by stress. There has been a concept that space traveling as a source of stress can alter the composition of the intestinal microbiome, which may result in the transient or permanent overgrowth of pathogenic gut bacteria (23). Consistently, as shown in Fig. 4, we demonstrated that gene expression of E. coli increased in HU group, which provide evidence that simulated microgravity lead to overgrowth of potential pathogenic gut bacteria $E$. coli. Bacteria (such as $S$. typhimurium) grown in spaceflight exhibited global changes in gene expression characterized with increased virulence expression (24). Besides, microgravity rendered $E$. coli more adherent to an intestinal epithelial-like Caco-2 cell line and markedly increased the production of the heat-labile enterotoxin (25). Whether the pathogenic bacteria grew under simulated microgravity with increased virulence to host requires further research.

Adaptations to new selective pressures may lead to microbiota at a tissue site breaking through the traditional host and microbe boundaries. Usually, intestinal microbiota and its human host coexist in good health and with mutual benefit. These commensal microbiota seldom cause disease except in rare cases such as in immunocompromised hosts or where the normal gastrointestinal barriers are breached (26). To explore the possibility that the overgrowth of some bacteria under microgravity circumstances can overcome epithelial barriers to initiate inflammation, we detected the endotoxin level in the blood. LPS, the major component of endotoxin, can bind to Toll-like receptor 4 (TLR4), initiating a potent cytokine cascade and inflammatory reaction. Unsurprisingly, we observed an increase in both LPS and LBP in the microgravity rat group. This was consistent with previous evidence indicating that changes in the dominant communities of microbiota compromise the host resistance to infection challenge by intestinal pathogens and predisposes the invasion of bacteria from the lumen to the interior body (23). Although E. coli is an important member of the normal intestinal microflora of humans and other mammals, this bacteria cause diseases in immunocompromised hosts or where the normal gastrointestinal barriers are breached (27). It have been reported that the expressions of hundred genes of $E$. coli were significantly altered in simulated microgravity conditions compared to that of normal gravity conditions (28). Together with the great changes in number of $E$. coli as shown in this study, we speculated that $E$. coli largely contributed to the increased level of endotoxin in the blood. The great changes of E. coli as observed under simulated microgravity may have huge influence on the health.

Changes in the diversity and number of gut microflora have been linked to immunological dysregulation, which is associated with many human noninfectious diseases such as autoimmunity, allergies and cancer (26). Indeed, simulated microgravity has recently been shown to increases susceptibility to colitis in mice (5). Sex hormones influence the development of autoimmune diseases (29). As we known, females suffer a higher incidence of many major autoimmune diseases and undergo changes in disease severity during pregnancy (29). Studies have recently linked this sexual dimorphism with the gut microbiota (30). For example, the most prominent case is microbiota in the development of autoimmune disease of Type 1 diabetes (T1D) in non-obesity diabetes (NOD) mice (30). Germ-free NOD males and females have been observed to develop T1D with a similar incidence. Moreover, male and female NOD mice suffer different T1D incidence after puberty with different intestine microbiota profiles (30). This indicates that sex hormone-mediated microbiota differences at least partially lead to variations in susceptibility to T1D. Based on all those results, we speculated that estrogen, the most well-known sex hormone, might counteract microgravity-induced gut flora changes and maintain intestinal mucosa homeostasis. As demonstrated in Fig. 4 that (HU+E4) and (HU+E8) groups with lower E. coli gene expresssion than HU group, it was agreed with our speculation that estrogen reversed microgravity-induced gut microbiota changes, especially inhibited the overgrowth of $E$. coli in the intestine of male rat under microgravity. Both the biodiversity index and level of serum LPS recovered to approximately normal when estrogen was administered to the blood, which also suggested a protective role for estrogen in maintaining the homeostasis of intestine mucosa. Also, we observed estrogen inhibited the overgrowth of B. fragilis and $F$. nucleatumis under microgravity. It have been reported that invasion of those bacteria are involved in the pathogenesis of colitis (31). So estrogen may decrease the susceptibility of autoimmune diseases such as colitis through regulation gut microbiota under microgravity.

Changes in the diversity and number of gut microflora, including reductions in the defense group of microorganisms and the overgrowth of opportunistic pathogens, such as E. coli, $B$. fragilis and $F$. nucleatumis, may profoundly influence health over the short and long term. The adapted bacteria clones under microgravity acquire specific virulence attributes, which confers an increased ability to evade the host defense and increase risk of many infectious and noninfectious diseases (32-34). Another concern is antibiotic resistance in bacteria under microgravity (35). It has reported that the minimum inhibitory concentration (MIC) of both colistin and kanamycin sharply increased in $E$. coli grown on a spaceflight module compared with that on the ground (35). This presents great challenges in preventing and controlling infection under microgravity. A better understanding of estrogen-microbiome crosstalk not only help in determining additional mechanisms involved in the pathogenesis of hormone-related diseases and may provide further evidences that administration of estrogen as a countermeasure to prevent intestinal microbiota-related disease during space flight missions.

\section{Acknowledgements}

The present study was supported by the China Scholarship Council (201400930009) and Beijing Natural Science Foundation (7162207). 


\section{References}

1. Saei AA and Barzegari A: The microbiome: The forgotten organ of the astronaut's body-probiotics beyond terrestrial limits. Future Microbiol 7: 1037-1046, 2012.

2. Cervantes JL and Hong BY: Dysbiosis and immune dysregulation in outer space. Int Rev Immunol 35: 67-82, 2016.

3. Tou JC, Grindeland RE and Wade CE: Effects of diet and exposure to hindlimb suspension on estrous cycling in Sprague-Dawley rats. Am J Physiol Endocrinol Metab 286: E425-E433, 2004.

4. Neuman H, Debelius JW, Knight R and Koren O: Microbial endocrinology: The interplay between the microbiota and the endocrine system. FEMS Microbiol Rev 39: 509-521, 2015

5. Li P, Shi J, Zhang P, Wang K, Li J, Liu H, Zhou Y, Xu X, Hao J, Sun X, et al: Simulated microgravity disrupts intestinal homeostasis and increases colitis susceptibility. FASEB J 29: 3263-3273, 2015

6. Ritchie LE, Taddeo SS, Weeks BR, Lima F, Bloomfield SA, Azcarate-Peril MA, Zwart SR, Smith SM and Turner ND: Space environmental factor impacts upon murine colon microbiota and mucosal homeostasis. PLoS One 10: e0125792, 2015.

7. Zhou Y, Ni H, Li M, Sanzari JK, Diffenderfer ES, Lin L, Kennedy AR and Weissman D: Effect of solar particle event radiation and hindlimb suspension on gastrointestinal tract bacterial translocation and immune activation. PLoS One 7: e44329, 2012

8. Looijer-van Langen M, Hotte N, Dieleman LA, Albert E, Mulder C and Madsen KL: Estrogen receptor- $\beta$ signaling modulates epithelial barrier function. Am J Physiol Gastrointest Liver Physiol 300: G621-G626, 2011

9. Menon R, Watson SE, Thomas LN, Allred CD, Dabney A, Azcarate-Peril MA and Sturino JM: Diet complexity and estrogen receptor $\beta$ status affect the composition of the murine intestinal microbiota. Appl Environ Microbiol 79: 5763-5773, 2013

10. Wang MH and Achkar JP: Gene-environment interactions in inflammatory bowel disease pathogenesis. Curr Opin Gastroenterol 31: 277-282, 2015.

11. Walter J and Ley R: The human gut microbiome: Ecology and recent evolutionary changes. Annu Rev Microbiol 65: 411-429, 2011.

12. Benson AK, Kelly SA, Legge R, Ma F, Low SJ, Kim J, Zhang M, Oh PL, Nehrenberg D, Hua K, et al: Individuality in gut microbiota composition is a complex polygenic trait shaped by multiple environmental and host genetic factors. Proc Natl Acad Sci USA 107: 18933-18938, 2010.

13. Du F, Ding Y, Zou J, Li Z, Tian J, She R, Wang D, Wang H, Lv D and Chang L: Morphology and molecular mechanisms of hepatic injury in rats under simulated weightlessness and the protective effects of resistance training. PLoS One 10: e0127047, 2015.

14. Zhang R, Ran HH, Cai LL, Zhu L, Sun JF, Peng L, Liu XJ, Zhang LN, Fang Z, Fan YY and Cui G: Simulated microgravity-induced mitochondrial dysfunction in rat cerebral arteries. FASEB J 28: 2715-2724, 2014

15. Su Y, Yao W, Perez-Gutierrez ON, Smidt H and Zhu WY: $16 \mathrm{~S}$ ribosomal RNA-based methods to monitor changes in the hindgut bacterial community of piglets after oral administration of Lactobacillus sobrius S1. Anaerobe 14: 78-86, 2008.

16. Breton J, Massart S, Vandamme P, De Brandt E, Pot B and Foligné B: Ecotoxicology inside the gut: Tmpact of heavy metals on the mouse microbiome. BMC Pharmacol Toxicol 14: 62, 2013

17. Lozupone CA and Knight R: Species divergence and the measurement of microbial diversity. FEMS Microbiol Rev 32: 557-578, 2008

18. Xu M, Wang B, Fu Y, Chen Y, Yang F, Lu H, Chen Y, Xu J and Li L: Changes of fecal Bifidobacterium species in adult patients with hepatitis B virus-induced chronic liver disease. Microb Ecol 63: 304-313, 2012.
19. Chen Y, Yang F, Lu H, Wang B, Chen Y, Lei D, Wang Y, Zhu B and Li L: Characterization of fecal microbial communities in patients with liver cirrhosis. Hepatology 54: 562-572, 2011.

20. Du Plessis J, Vanheel H,Janssen CE, Roos L, Slavik T, Stivaktas PI, Nieuwoudt M, van Wyk SG, Vieira W, Pretorius E, et al: Activated intestinal macrophages in patients with cirrhosis release $\mathrm{NO}$ and IL-6 that may disrupt intestinal barrier function. J Hepatol 58: $1125-1132,2013$

21. Barman M, Unold D, Shifley K, Amir E, Hung K, Bos N and Salzman N: Enteric salmonellosis disrupts the microbial ecology of the murine gastrointestinal tract. Infect Immun 76: 907-915, 2008.

22. Lynch SV, Brodie EL and Matin A: Role and regulation of sigma $\mathrm{S}$ in general resistance conferred by low-shear simulated microgravity in Escherichia coli. J Bacteriol 186: 8207-8212, 2004

23. Bailey MT, Dowd SE, Galley JD, Hufnagle AR, Allen RG and Lyte M: Exposure to a social stressor alters the structure of the intestinal microbiota: Implications for stressor-induced immunomodulation. Brain Behav Immun 25: 397-407, 2011.

24. Rosenzweig JA, Abogunde O, Thomas K, Lawal A, Nguyen YU, Sodipe A and Jejelowo O: Spaceflight and modeled microgravity effects on microbial growth and virulence. Appl Microbiol Biotechnol 85: 885-891, 2010.

25. Allen CA, Niesel DW and Torres AG: The effects of low-shear stress on Adherent-invasive Escherichia coli. Environ Microbiol 10: 1512-1525, 2008.

26. Zhang YJ, Li S, Gan RY, Zhou T, Xu DP and Li HB: Impacts of gut bacteria on human health and diseases. Int J Mol Sci 16: 7493-7519, 2015

27. Gombošová L, Lazúrová I, Zakuciová M, Curová K, Kmet'ová M, Petrášová D and Siegfried L: Genes of intestinal Escherichia coli and their relation to the inflammatory activity in patients with ulcerative colitis and Crohn's disease. Folia Microbiol (Praha) 56: 367-372, 2011.

28. Arunasri K, Adil M, Venu Charan K, Suvro C, Himabindu Reddy S and Shivaji S: Effect of simulated microgravity on E. Coli K12 MG1655 growth and gene expression. PLoS One 8: e57860, 2013

29. Rubtsova K, Marrack P and Rubtsov AV: Sexual dimorphism in autoimmunity. J Clin Invest 125: 2187-2193, 2015.

30. Yurkovetskiy L, Burrows M, Khan AA, Graham L, Volchkov P, Becker L, Antonopoulos D, Umesaki Y and Chervonsky AV: Gender bias in autoimmunity is influenced by microbiota. Immunity 39: 400-412, 2013.

31. Xavier RJ and Podolsky DK: Unravelling the pathogenesis of inflammatory bowel disease. Nature 448: 427-434, 2007.

32. Bashir A, Miskeen AY, Bhat A, Fazili KM and Ganai BA: Fusobacterium nucleatum: An emerging bug in colorectal tumorigenesis. Eur J Cancer Prev 24: 373-385, 2015.

33. Sears CL, Geis AL and Housseau F: Bacteroides fragilis subverts mucosal biology: From symbiont to colon carcinogenesis. J Clin Invest 124: 4166-4172, 2014.

34. Castellarin M, Warren RL, Freeman JD, Dreolini L, Krzywinski M, Strauss J, Barnes R, Watson P, Allen-Vercoe E, Moore RA and Holt RA: Fusobacterium nucleatum infection is prevalent in human colorectal carcinoma. Genome Res 22: 299-306, 2012

35. Mermel LA: Infection prevention and control during prolonged human space travel. Clin Infect Dis 56: 123-130, 2013.

This work is licensed under a Creative Commons

Attribution-NonCommercial-NoDerivatives 4.0 International (CC BY-NC-ND 4.0) License. 